\title{
Norois
}

Environnement, aménagement, société

$203 \mid 2007 / 2$

Mobilité, hydrologie, loisir et tourisme, espace urbain

\section{Renouvellement urbain à Brest : les enseignements du marché de définition du plateau des Capucins}

Urban renewal in Brest: the learnings of the new project of the "plateau des Capucins"

Pierre-Arnaud Barthel

\section{CpenEdition}

Journals

Édition électronique

URL : https://journals.openedition.org/norois/1508

DOI : $10.4000 /$ norois. 1508

ISBN : 978-2-7535-1551-2

ISSN : $1760-8546$

Éditeur

Presses universitaires de Rennes

Édition imprimée

Date de publication : 1 juin 2007

Pagination : 7-25

ISBN : 978-2-7535-0511-7

ISSN : 0029-182X

Référence électronique

Pierre-Arnaud Barthel, « Renouvellement urbain à Brest : les enseignements du marché de définition du plateau des Capucins », Norois [En ligne], 203 | 2007/2, mis en ligne le 01 juin 2009, consulté le 13 janvier 2022. URL : http://journals.openedition.org/norois/1508; DOl : https://doi.org/10.4000/norois. 1508

(c) Tous droits réservés 


\title{
Renouvellement uRBAin À Brest : LES ENSEIGNEMENTS \\ DU MARCHÉ DE DÉFINITION DU PLATEAU DES CAPUCINS
}

\author{
Pierre-Arnaud BARTHEL \\ CEsTAN - UMR 6590 CNRS Espaces et SociéTÉS \\ (Université de Nantes), \\ rue de la Censive-du-Tertre - 44300 NANTES \\ pierre-arnaud.barthel@univ-nantes.fr
}

\section{RÉSUMÉ}

La mise en projet du plateau des Capucins, en cours de cession par la Marine à la communauté urbaine de Brest, est l'objet de cet article. Le temps de la procédure du marché de définition est un moment privilégié d'observation qui permet au chercheur d'éclairer les modalités et épreuves $d u$ portage politique et $d u$ pilotage technique, les lieux et scènes de négociation/participation où se joue le projet sur des enjeux et «dossiers » très variés (identité urbaine, attractivité territoriale, couplage avec le projet de tramway et stratégie métropolitaine). In fine, le surinvestissement des acteurs locaux depuis 2004 sur ce fragment de ville projetée est l'occasion de mettre au jour comment se fabrique actuellement un "grand projet », reconnu et labellisé comme tel en France au-delà de la sphère locale. Au regard d'autres projets urbains en cours sur des friches situées dans les grandes villes de l'Ouest français, qui misent sur l'économie culturelle, les pôles de compétences et l'événementiel, cette étude conduit en conclusion à faire état d'un certain nombre de convergences et circulations dans les modes de faire de l'aménagement.

MotS CLÉS : Brest - Économie de la connaissance - Friches militaires - Innovation - Projet urbain - Renouvellement urbain.

\section{ABSTRACT}

\section{Urban renewal in Brest: the learnings of the new project of the "plateau des} Capucins"

This article mainly deals with the new urban project of the "plateau des Capucins", which the Marine is in the course of transferring to the urban community of Brest. The time of the proceeding of "marché de definition" (city-planning teams in competition) is a great moment to focus on the modalities and difficulties of political and communicational leading; the places and scenes of public negotiation/participation on several subjects (such as urban identity, territorial attractiveness and metropolitan project); and finally the tensions linked to the over-investment of many actors involved in this urban renewal. This newly-born action provides an opportunity to disclose the way a "big project" is nowadays built in France - recognized and labeled as such - beyond the local sphere. In the context of the urban regeneration in the West of France, the conclusion explores the models in town-planning shared by the big cities of this area.

KEY WORDS : Brest - Innovation - Knowledge Economy - Military Brown Fields Urban Project - Urban Renewal. 
Encore plein d'incertitudes dans son montage opérationnel en 2005, un grand projet urbain est né à Brest, action urbanistique qui pourrait contribuer à l'accession au rang métropolitain de cette agglomération située à l'extrémité ouest de la France. Le site du projet est un promontoire de 12 ha au-dessus de la rivière Penfeld situé en face du centre-ville reconstruit, que la Marine nationale n'utilise plus depuis 2004 pour cause de restructuration des activités de l'arsenal. Certes la superficie engagée n'est pas comparable aux 340 ha de l'Île de Nantes (située à Nantes), ni au périmètre du projet Ville-Port de Saint-Nazaire, mais ce serait une erreur de ne considérer que le plateau strictosensu. En effet, le projet naissant prend place dans un ensemble de trois cadres d'action structurants. Tout d'abord, la construction d'une vision stratégique pour le (re)développement du front de mer de Brest (plusieurs ZAC en cours) est formalisée depuis 2000 (Adeupa, 2003). Ensuite, la mutation de la Penfeld est pensée simultanément sur trois sites (fig. 1) conformément au CIADT de mai 2000 : les Capucins, mais aussi le port du Château (en phase opérationnelle depuis 2005) et le fond de Penfeld (le Salou en phase d'études pré-opérationnelles). Enfin, la planification stratégique par le biais du Projet d'Aménagement et de Développement Durable (intégrée au Plan Local de l'Urbanisme de Brest) est un troisième document-cadre qui intègre le projet des Capucins.

Suite à l'annonce de DCN-Brest (Direction des Chantiers Navals) de déménager des ateliers historiques des Capucins et de regrouper ses activités au bord de la rade de Brest, Brest Métropole Océane $(\mathrm{BMO})^{l}$ s'est saisi de l'opportunité pour engager une réflexion sur l'avenir du site. Et le mois d'octobre 2005 a clôturé le marché de définition lancé en mars 2004 par la collectivité à propos du renouvellement urbain du plateau des Capucins. Cet article est l'occasion de revenir sur le temps de cette procédure d'aménagement qui a constitué un grand moment pour le milieu urbanistique local (technique et politique). Il est fondateur au moins à triple titre. Le marché de définition est tout d'abord la concrétisation d'un mode projet qui se formalise en même temps que se joue un événement majeur : la constitution de BMO comme maître d'ouvrage urbain avec toutes les difficultés (opérationnelles, humaines, techniques, programmatiques et financières) que cela implique pour cette intercommunalité dans le jeu d'acteurs qui se structure à cette occasion. Deuxième temps de la réflexion, le marché de définition est une véritable entrée en scène $(\mathrm{s})$ du plateau des Capucins qui était jusque-là un cœur de ville interdit aux Brestois. Murs d'enceinte, portes d'accès réservées à la Marine et falaises ont empêché toute forme d'appropriation sociale, exception faite des « Portes ouvertes » de 2004 et de 2005 pendant lesquelles les déambulations ont été autorisées. Dans ce contexte particulier, «l'identité brestoise » est convoquée par les acteurs et (re)négociée à travers le projet. Dernier temps de cet article, le marché de définition traduit a priori un urbanisme concerté entre les acteurs de l'Etat et locaux, mais qui n'est pas exempt de vives confrontations au point de soupçonner en définitive le discours convenu sur la « vision partagée » à laquelle la mise en œuvre de la procédure aurait naturellement abouti. Replacé dans le contexte actuel des projets de renouvellement urbain des grandes villes de l'Ouest français, le cas brestois donnera à penser pour appréhender des convergences dans les modes contemporains de « faire la ville sur la ville».

Au plan méthodologique, le cadre d'analyse de cette recherche conduite entre mai 2005 et octobre 2006 emprunte au «tournant pragmatique » (Thévenot, 2006) et vise à regarder les acteurs au travail ${ }^{2}$. L'enjeu est de documenter les épreuves de concrétisation de ce nouveau projet à travers un certain nombre de documents techniques d'urbanisme ${ }^{3}$ (document-programme, cahier des charges...), de supports de la communication officielle (sites Internet, plaquettes...), de comptes-rendus de réunions internes, de réunions publiques observées et d'entretiens réalisés

1. La communauté urbaine de Brest s'est rebaptisée depuis janvier 2005 «Brest Métropole Océane ».

2. Cet article est nourri d'une méthodologie partagée par une équipe de chercheurs des laboratoires du LAUA et de l'UMR ESO dans le cadre d'une recherche-action conduite sur une période de trois ans (2003-2007), en parallèle à ce travail, sur la fabrique urbanistique nantaise (L. Devisme, directeur scientifique) - Plate-forme d'Observation des Projets et Stratégies Urbaines (POPSU).

3. Certains des documents consultés étaient confidentiels. Nous remercions les acteurs locaux qui ont bien voulu nous faire confiance. 
avec quelques acteurs du projet. Chercher à élucider l'action collective conduit à privilégier des prismes d'analyse : les scènes plus ou moins confidentielles et emboîtées où se jouent le projet et qui construisent un parcours avec ses lieux obligés pour devenir un « grand projet », les temporalités et leur lot d'incertitudes pour BMO, et le travail des acteurs sur un certain nombre de dossiers à enjeux (le devenir des ateliers, le tracé du tramway, la définition d'un parcours et d'un centre d'interprétation).

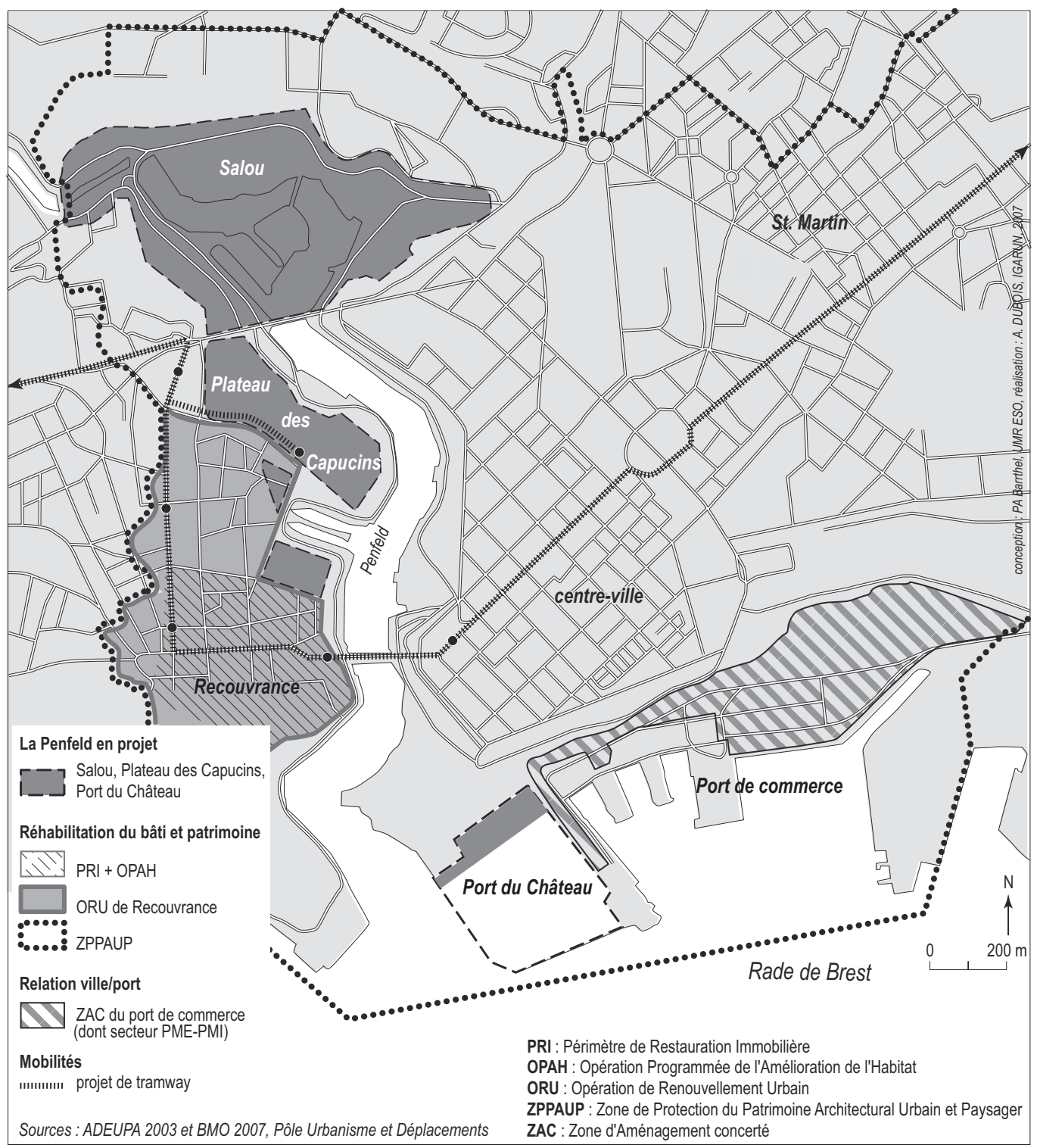

Figure 1 : Le renouvellement urbain brestois : quartiers centraux, façade portuaire et Penfeld (source : BMO, 2007) 


\section{Le marché de définition comme épreuve de concrétisation à double détente}

Le marché de définition confirmé par le dernier Code des marchés publics est une procédure qui connaît un grand succès depuis 1996. Le recours à ce mode de faire est croissant par rapport à d'autres modes de consultation plus classiques tels que les concours d'idées et autres consultations directes. Elle est rapidement justifiée par le maire de Brest et président de BMO au cours de la première réunion publique de présentation à Brest des projets en juin 2005 : "La nature du site, sa localisation en cœur de ville, son intérêt patrimonial, nous ont amenés à engager une démarche originale : un "marché de définition". Il vise, non pas à choisir, par concours d'architecte, un projet "clé en main", mais à élaborer une sorte de "plan guide" pour l'aménagement futur de l'espace » (F. Cuillandre).

\section{RÉ-APPROPRIATION LOCALE D'UN SITE « INTERDIT » ET CONSTITUTION D'UNE MAÎTRISE D'OUVRAGE URBAINE}

Le marché de définition peut se lire comme une épreuve de concrétisation du projet à double détente : une phase ouverte au cours de laquelle un programme a été co-construit sans mise en concurrence entre les équipes d'urbanistes sélectionnées suite à l'appel d'offres du marché et sur un mode itératif avec le commanditaire; une phase fermée au cours de laquelle les équipes sont en concurrence et doivent proposer leur vision opérationnelle et stratégique sur la base du préprogramme validé à la fin de la phase ouverte (SEMAEB/BMO, 2005). De l'avis des professionnels et de certains chercheurs en aménagement, l'avantage est d'ouvrir une concertation très en amont dès la phase ouverte de discussion collective entre le commanditaire et les équipes du marché. Ces dernières, constituant une sorte de maîtrise d'ouvrage bis collective élargie aux partenaires institutionnels, aident élus et professionnels à donner du sens au projet et préparent les conditions de l'attribution du marché de maîtrise d'œuvre. Toutefois les inconvénients ont d'ores et déjà été repérés : la procédure pallie à la faiblesse d'une maîtrise d'ouvrage en manque d'idées et de vision stratégique (Zetlaoui-Leger, 2002). À Brest, les acteurs locaux, dont Annick Cléach, Première viceprésidente de $\mathrm{BMO}$, portent un jugement ex post très positif de cette procédure perçue comme innovante au moment de sa mise en place (d'après entretien réalisé en juin 2007).

Ce moment pré-opérationnel s'est traduit par la formalisation d'un jeu d'acteurs, sans qu'il soit pour autant à ce jour complètement stabilisé. La structuration est double. À un premier niveau, en amont du marché de définition, une « mission Penfeld ${ }^{4}$ » est lancée pour les études et le suivi de la mise en projet des terrains dont la cession par la Marine est potentiellement envisageable. Elle fait suite au CIADT du 18 mai 2000 et à la réunion interministérielle du 5 mars $2002^{5}$ : l'État envoie alors un chargé de mission pour fédérer le milieu local - en la personne de G. de Senneville, inspecteur général du ministère de l'Équipement. Le plan de charge de ce dernier, qui a rendu son rapport clôturant son travail en 2005, tient en deux actions dépendantes l'une de l'autre : d'une part, proposer un ensemble cohérent de décisions permettant d'engager une première opération, qui est la transformation du port du Château en un port d'escale pour la navigation de plaisance, d'autre part, présenter un schéma d'évolution du site de la Penfeld faisant l'objet d'un consensus entre l'État et la communauté urbaine de Brest (De Senneville, 2005). L'enjeu n'est pas des

4. La Mission Penfeld est coprésidée par le président de la Communauté urbaine de Brest et le préfet du Finistère. Les membres émanent des institutions centrales (DIRD, DATAR, MRAI, ABF ...), régionales et départementales (SGAR, DDE, conseils général et régional) et locales (élus de la CUB, CCI, DCN Brest).

5. D'après le dossier de presse du CIADT du 5 mars 2002, depuis 1997, le re-dimensionnement de la marine nationale et la restructuration des industries de défense ont entraîné une mutation rapide de la structure économique et sociale du pays de Brest. Pour accompagner cette mutation, l'État et les collectivités ont décidé de renforcer les pôles de compétences brestois. Les mesures décidées lors des CIADT précédents ont permis de développer le pôle « mer » avec les travaux sur la création de l'institut d'ingénierie des sciences de la mer, et la création d'un pôle à vocation nationale sur l'aménagement du littoral, l'environnement côtier et l'océanographie côtière. 
moindres : faire se rencontrer des mondes professionnels qui s'étaient jusque-là peu fréquentés, d'un côté, la Marine et son ministère de tutelle, et de l'autre, le milieu local brestois des élus et des services de la ville et de la communauté urbaine; bref à l'occasion du désengagement de la Marine sur des sites identifiés, résoudre une tension État/local, soit une recomposition des relations entre l'institution militaire et les décideurs urbains vers des accords négociés sur le devenir de la Penfeld.

La constitution d'une maîtrise d'ouvrage urbaine à l'occasion du marché de définition est le second niveau de la mise en place du jeu d'acteurs. L'épreuve du portage politique du futur projet découle de cette désignation décisive. La mise en concurrence de l'État face aux collectivités locales est très vite écartée, la constitution de la mission Penfeld ayant déminé le problème et légitimé par avance la (ré)appropriation de ce territoire par les locaux. En revanche, se pose l'alternative d'un portage communal ou intercommunal pour ces terrains situés stratégiquement à proximité du centre-ville reconstruit. L'échelle du portage politique est cruciale et détermine par avance la captation des financements et le niveau d'ambition auquel les acteurs locaux se hissent pour ce territoire à renouveler. En 2004, les élus ont vite tranché en faveur de l'intercommunalité dont le besoin en projets s'est avéré un argument clé de la décision. En s'entourant de bureaux d'études en guise d'assistance à maîtrise d'ouvrage, cette dernière se place de facto au cœur du pilotage du marché de définition dont les études ont été financées par BMO elle-même, le FRED ${ }^{6}$ et le FEDER. De plus, BMO prend l'initiative de réunions thématiques hors marché de définition sur des options diverses et décisives : discuter de la commande d'un parcours et d'un centre d'interprétation, ou encore engager des enquêtes auprès des professionnels pour affiner la programmation bureaux ou bien celle en logements. Dernier niveau d'action pour BMO, les actions de communication et de participation des habitants donnent lieu à la construction d'un espace Internet dédié à la Penfeld, à des réunions publiques en lien avec le marché de définition, à des journées portes ouvertes et des temps pour la présentation des projets des trois équipes d'urbanistes de renom retenues au début de la procédure (Fortier, Reichen et Robert, et Madec).

\section{UNE PHASE OUVERTE POUR SE METTRE D'ACCORD SUR UN PROGRAMME}

Le périmètre d'étude d'une superficie de 16 ha comprend les ateliers des Capucins, libérés par DCN-Brest fin 2004 et remis à l'État; le plateau de Quéliverzan, qui comprend des bâtiments encore utilisés par la Marine mais qui peuvent fonctionner ailleurs dans un environnement civil ou non, la prison de Pontaniou, propriété de BMO, et ses alentours (le bas de la rue de Saint-Malo, le bâtiment aux lions et le terrain de la Madeleine $)^{7}$. Le diagnostic territorial (CUB, 2003) a naturellement mis en valeur tout le potentiel de cette "presqu'île » en plein cœur de la ville, véritable « belvédère » au riche patrimoine industriel et architectural dont certains bâtiments sont d'ailleurs protégés au titre de la ZPPAUP ${ }^{8}$ (notamment la prison de Pontaniou et l'atelier d'électricité, mais aussi les quais et fortifications) ou des Monuments historiques (comme dans le cas des Ateliers des machines ou du bâtiment aux Lions). En revanche le plateau de Quéliverzan recèle moins de merveilles patrimoniales : centre de formation, restaurant et installations sportives réservés à l'arsenal y sont regroupés. Le point noir du site est son enclavement relatif. D’un côté, un haut mur d'enceinte long de 530 m coupe le double plateau Capucins/Quéliverzan du quartier faubourien de Recouvrance (qui fait l'objet d'une Opération de Renouvellement Urbain), de l'autre, une falaise

6. Pour atténuer l'impact des mesures de restructuration militaire et les effets de l'évolution de l'industrie de l'armement, le gouvernement a affecté une enveloppe financière spécifique. Le fonds de restructuration de la défense (FRED) est en place depuis 1991. Il est réservé aux bassins d'emploi touchés par la réduction du format des armées et/ou par la baisse des activités industrielles ou des effectifs du secteur de l'armement. Destinées à renforcer le tissu économique local, les subventions du FRED sont attribuées à des projets variés et soutiennent plusieurs types de bénéficiaires.

7. Le périmètre de réflexion comprend en outre le terrain des casernes qui fait partie et restera dans le domaine militaire, mais que la Marine souhaite voir intégrer dans leur environnement immédiat.

8. Zone de Protection du Patrimoine Architectural, Urbain et Paysager (ZPPAUP). 
sur près de 730 m suspend ce plateau privé d'accès en contrebas au niveau des quais qui resteront la propriété de la Marine. Le pont de l'Harteloire à l'extrémité nord du plateau de Quéliverzan est un point d'articulation avec le reste des quartiers (fig. 2 et photo 1).

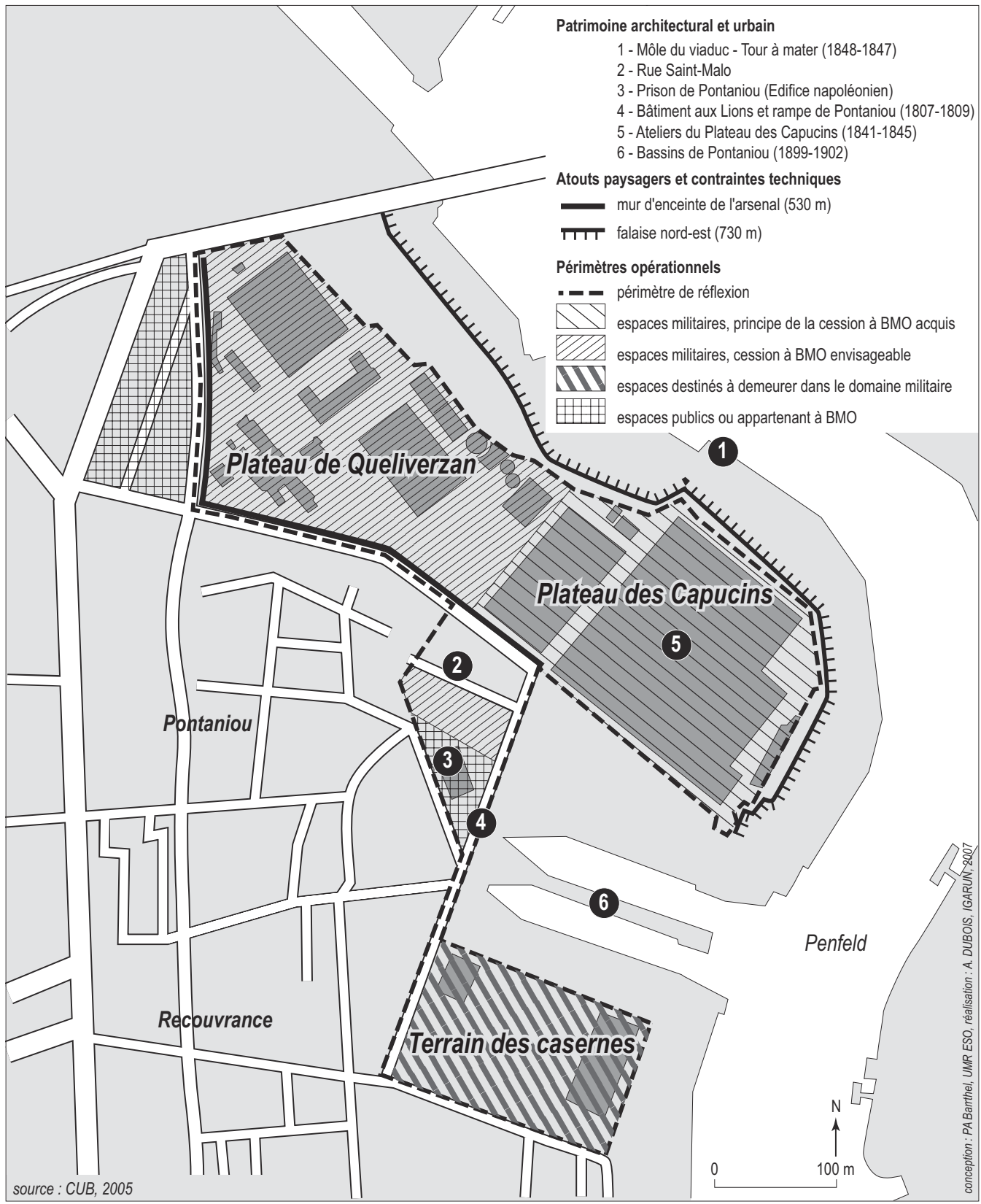

Figure 2 : Le plateau des Capucins à Brest, patrimoine et périmètres de projet (situation en 2005) (source : CUB, 2005)

Heritage and location of the perimeter of the preliminary studies 


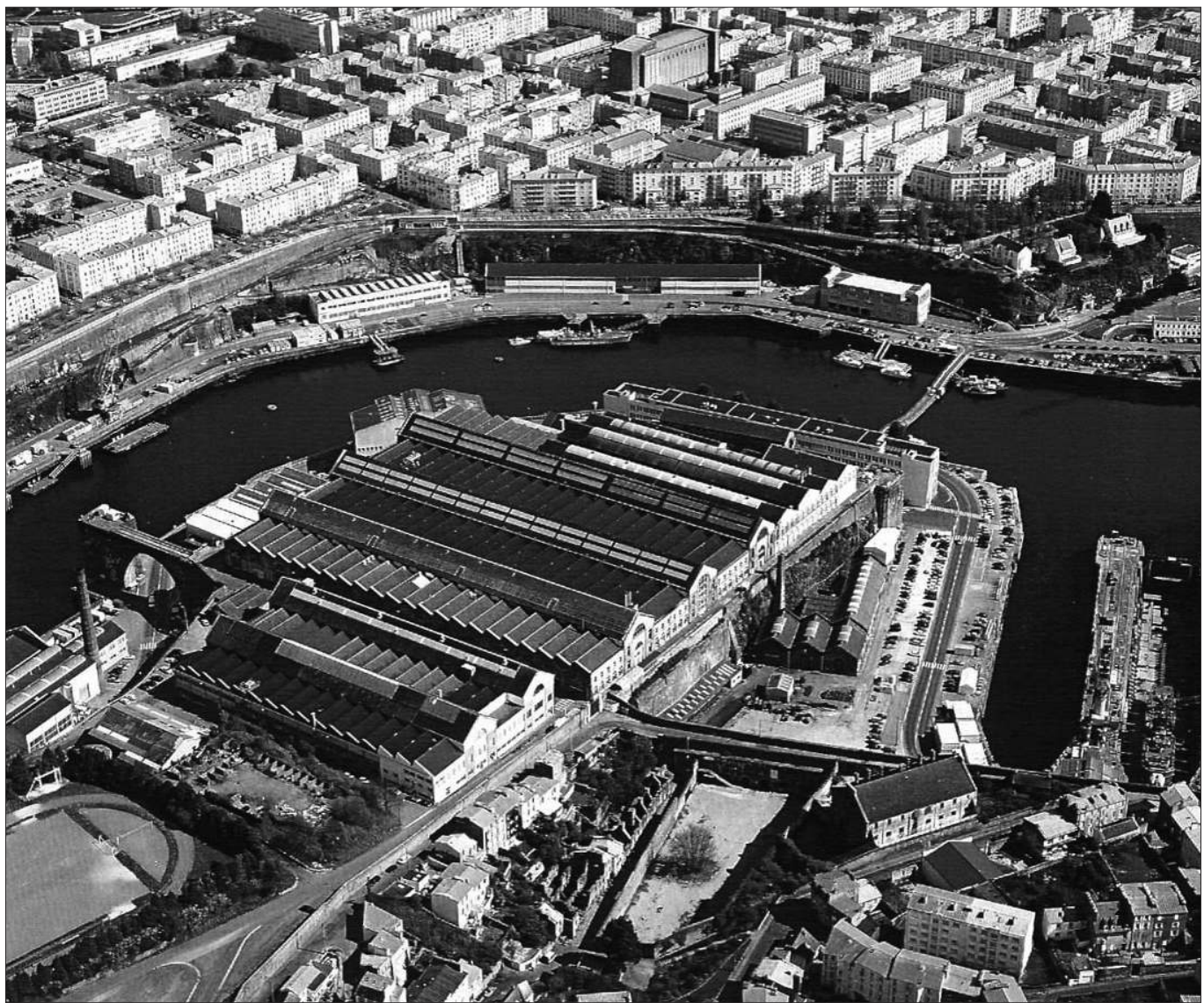

Photo 1 : Vue aérienne du territoire de projet (source : CUB, 2005) Overview of the project territory

Les attendus de cette phase ouverte sont de «faire émerger des éléments de programme et des contenus en termes de concepts spécifiques de l'identité brestoise » (CUB, 2004). Le documentprogramme remis par le maître d'ouvrage aux trois équipes de maîtrise d'œuvre à l'issue de la phase collective du marché de définition est un texte de première importance en ce qu'il stabilise la commande et reflète un certain nombre de décisions programmatiques qui ont été validées par le comité de pilotage. Préparé en amont dans le cadre des réunions pilotées par la Mission Penfeld ${ }^{9}$, l'accord s'est ainsi confirmé autour de l'objectif de fabriquer un nouveau quartier pour Brest dont la fonction emblématique doit être lisible et visible. Parce que le registre de production n'est clairement pas celui de la fabrication ordinaire de la ville, plusieurs pistes ont été identifiées pour asseoir l'exemplarité de ce territoire à renouveler. Tout d'abord, le positionnement sur des pôles de compétitivité existants ou à conforter afin de valoriser les savoir-faire brestois : le tertiaire haut de gamme lié à la mer et au littoral et la «filière » image et son (France 3 Iroise, pépinière d'entreprises). Aux bureaux programmés s'ajoutent les espaces d'exposition, de manifestations événementielles, d'hôtellerie et de séminaires. Ensuite, le levier culturel est clairement convoqué et se formalise par la création d'une « congestion » d'équipements publics dans les nefs des ateliers des Capucins, fruit

9. La réunion du 14 janvier 2003 des membres de la mission Penfeld a fixé un certain nombre d'orientations avec les élus la volonté a été unanime pour faire des Capucins un vrai quartier de ville avec, entre autres, l'idée d'une trame verte et la nécessité d'un certain nombre d'équipements structurants. 
de stratégies de relocalisations/transferts de structures culturelles déjà existantes sur Brest (suite à contacts, des acteurs locaux sont déjà positionnés sur l'échiquier du projet : école des Beaux-Arts, Cinémathèque de Bretagne, le Fourneau - un pôle associatif du théâtre vivant...) et de création de nouveaux équipements (résidence et maison internationales, ateliers et logements d'artistes). Le programme habitat, quant à lui, reflète l'objectif de 350 logements ${ }^{10}$ (sans plus de précision dans le document-programme) et une offre a minima (pas plus de $20 \%$ ) de logements aidés (sociaux et abordables), compte tenu du profil dominant d'habitat social des quartiers riverains (Recouvrance et grand ensemble de Quéliverzan). Enfin, l'une des clés du projet est de concilier (à force d'imbrications des fonctions et de mutualisations des espaces et autres stationnements) le parti d'un quartier d'affaires liées à la mer et la fonction résidentielle. Il recèle une tension entre ces deux objectifs dans la mise en œuvre non soulevée par le maître d'ouvrage ${ }^{11}$.

Une différenciation interne du projet se lit en creux : la programmation bureaux/logements/commerces du plateau de Quéliverzan devrait tendre à équilibrer - par le biais des ventes de charges foncières - la restructuration des ateliers des Capucins qui s'annonce extrêmement complexe et coûteuse. Au fil des pages du document-programme (CUB, 2004), les principes idéologiques s'égrènent : " mixité et qualité résidentielle », "parti urbain compact », " démarche contextualisée » (prise en compte des dimensions paysagère, historique et sociale), « les modes doux à l'honneur », «faire lien ». Rien n'est oublié jusqu’aux prescriptions environnementales : «prendre en compte le climat local », « réaliser des économies d'énergie et maîtriser les rejets ». La disposition d'un certain nombre d'objets spatiaux, de dispositifs traduisant l'ambition, se joue à travers cette phase collective du marché de définition. Des chiffres « sortent » : il est question de $100000 \mathrm{~m}^{2}$ de Surface Hors Euvre Nette (SHON - dont 30000 pour les bureaux et 35000 pour les logements) et le montant prévisionnel des travaux est de 150 millions d'euros. Le projet de renouvellement urbain du plateau des Capucins se construit également en lien avec les autres projets « dans les tuyaux ». En outre, celui du tramway s'est défini parallèlement au marché de définition. Deux variantes de tracés aux abords du site du plateau étaient envisagées au démarrage du marché de définition : la première avec la possibilité d'un nouveau franchissement sur le fleuve, la seconde l'excluant. Très vite, BMO a validé la seconde option demandant aux urbanistes en concurrence d'exclure l'idée d'un nouveau pont pour le tramway.

\section{UNE PHASE FERMÉE : ForTIER GAGNANT}

À l’issue de la phase fermée, les trois équipes ont rendu leur projet formalisé à travers les différents documents techniques demandés : un "plan guide » proposant une vision à dix ans composé d'un plan stratégique d'ensemble, de coupes, d'images et autres schémas, une note de synthèse, un phasage d'intervention et une proposition de mission de maîtrise d'œuvre. Non seulement devant la commission d'appel d'offres, mais aussi au cours de réunions publiques très suivies par les Brestois au cours du mois de juin 2005, la révélation des trois projets a constitué un grand moment local. Et la désignation de l'équipe Fortier par le Bureau communautaire du 25 novembre 2005, sur proposition de la commission d'appel d'offres spécifique, pour concevoir et mettre en œuvre le projet a clôturé le marché de définition. Annick Cléach, Première Vice-présidente de BMO, a rappelé dans la presse locale les raisons de la réussite du projet Fortier : «Une vision globale du projet à l'échelle de l'agglomération, le respect des caractéristiques géographiques du site, une attention portée à la valeur symbolique du lieu et une utilisation intéressante des transports en commun pour bien irriguer le quartier » (Le Télégramme de Brest, 29 octobre 2005). De l'avis d'une chargée de mission en charge du dossier, architecte à l'Agence d'urbanisme de Brest, rencontrée

10. En 2007, l'objectif de 350 logements a été porté à 600 : « La programmation s'est affinée depuis la fin du marché de définition. Un parti plus clair en faveur de la densité s'est dessiné chemin faisant avec l'urbaniste lauréat Bruno Fortier. Et en interne, on a ajusté nos objectifs après beaucoup de discussions » (entretien réalisé avec Annick Cléach, juin 2007).

11. On retrouve pareille difficulté pour les acteurs locaux sur d'autres territoires comme «Euronantes Gare » (au sortir de la gare TGV) à Nantes, composante du Grand Projet de Ville « Malakoff-Pré Gauchet » (Dumont et Andrieu, 2006). 
en juin 2006, « cette équipe a emporté la mise sur différents points : meilleure prise en compte de l’insertion urbaine avec les quartiers riverains, bonne gestion des aspects symboliques (mémoire ouvrière et parcours d'interprétation)».

\begin{tabular}{|c|c|c|c|}
\hline Projet & quipe Fortier & Équipe Reichen et Robert & Équipe Madec \\
\hline $\begin{array}{l}\text { Composition } \\
\text { urbaine }\end{array}$ & $\begin{array}{l}\text { liers (quartier bas) et futur quar- } \\
\text { mixte (quartier haut) : } 2 \text { quartiers } \\
\text { arés }\end{array}$ & $\begin{array}{l}\text { Tripartition : pôle affaires au bord de } \\
\text { la rivière, quartier résidentiel et pôle } \\
\text { des ateliers }\end{array}$ & $\begin{array}{l}\text { Ordonnancement de part et d'autre } \\
\text { d'un axe central accueillant le tram }\end{array}$ \\
\hline $\begin{array}{l}\text { Philo } \\
\text { projet }\end{array}$ & $\begin{array}{l}\text { une mutation qui n'a pas à être } \\
\text { chée » }\end{array}$ & $\begin{array}{l}\text { Effet citadelle de la péninsule à pré- } \\
\text { erver » }\end{array}$ & $\begin{array}{l}\text { Approche envi } \\
\text { nisme }\end{array}$ \\
\hline $\begin{array}{l}\text { Approche envi- } \\
\text { ronnementale }\end{array}$ & $\begin{array}{l}\text { Pas vraiment abordée, pas de réfé- } \\
\text { rence au développement durable }\end{array}$ & $\begin{array}{l}\text { Pas vraiment abordée, récupération } \\
\text { des eaux pluviales envisagée }\end{array}$ & $\begin{array}{l}\text { Piles photovoltaïques, turbines, récu- } \\
\text { pération des eaux pluviales, végétali- } \\
\text { sation }\end{array}$ \\
\hline $\begin{array}{l}\text { Restructuration } \\
\text { des grands } \\
\text { ateliers }\end{array}$ & $\begin{array}{l}\text { Nef centrale dédiée à l'espace public. } \\
\text { Programme mixte: filière Image et } \\
\text { Son, espaces médias, séminaires et } \\
\text { congrès, équipements culturels, cen- } \\
\text { tre d'interprétation }\end{array}$ & $\begin{array}{l}\text { Diagonale percée et patio dans la } \\
\text { structure en nefs longitudinales. } \\
\text { Même programme mixte et bureaux } \\
\text { projetés également. }\end{array}$ & $\begin{array}{l}\text { Station de tram et parking dans les } \\
\text { ateliers } \\
\text { Programme mixte: équipements } \\
\text { publics dans les nefs, commerces } \\
\text { et hébergement (hôtellerie), filière } \\
\text { Image et Son, bureaux }\end{array}$ \\
\hline $\begin{array}{l}\text { Requ } \\
\text { de la } \\
\text { Pont }\end{array}$ & $\begin{array}{l}\text { licis } \\
\text { ech- }\end{array}$ & $\begin{array}{l}\text { ue : studios son et } \\
\text { n }\end{array}$ & $\begin{array}{l}\text { Centre d'interprétation de l'histoire } \\
\text { de Brest }\end{array}$ \\
\hline $\begin{array}{l}\text { Commerces } \\
\text { d'agglomération }\end{array}$ & $\begin{array}{l}\text { Concentration au débouché du pont } \\
\text { de l'Harteloire (limite nord) en lien } \\
\text { avec la station TCSP (visibilité et } \\
\text { accessibilité) }\end{array}$ & $\begin{array}{l}\text { Mixés avec le parking en silo dans } \\
\text { l'atelier d'électricité reconverti et inté- } \\
\text { grés aux ateliers }\end{array}$ & $\begin{array}{l}\text { Concentration au débouché du pont } \\
\text { de l'Harteloire (limite nord) en lien } \\
\text { avec la station TCSP (visibilité et } \\
\text { accessibilité) }\end{array}$ \\
\hline Habitat & $\begin{array}{l}\text { Mixité systématique à l'îlot avec la } \\
\text { fonction tertiaire. Majorité collectif : } \\
\mathrm{R}+4 / \mathrm{R}+6\end{array}$ & $\begin{array}{l}\text { Îlots dédiés (+commerces) localisés } \\
\text { en arrière des ilots bureaux. Typo- } \\
\text { logies variées: collectifs, maison de } \\
\text { ville et villas urbaines. }\end{array}$ & $\begin{array}{l}\text { Mixité possible à l'îlot avec la fonc- } \\
\text { tion tertiaire. Volumétrie dégressive, } \\
\text { typologies variées : collectifs, «villas } \\
\text { urbaines». }\end{array}$ \\
\hline $\begin{array}{l}\text { Fonction ter- } \\
\text { tiaire }\end{array}$ & $\begin{array}{l}\text { Mixité bureaux / habitat. Localisation } \\
\text { à l'îlot peu précise }\end{array}$ & $\begin{array}{l}\text { en front de Pen- } \\
\text { ers : vitrine d'af- }\end{array}$ & $\begin{array}{l}\text { Saupoudrage des activités de } \\
\text { dans les îlots et dans les ateli }\end{array}$ \\
\hline $\begin{array}{l}\text { Transition avec } \\
\text { les quartiers } \\
\text { Recouvrance et } \\
\text { Quéliverzan }\end{array}$ & $\begin{array}{l}\text { Traitée par la promenade et le recul } \\
\text { des nouveaux immeubles + Abaisse- } \\
\text { ment du mur }\end{array}$ & $\begin{array}{l}\text { Côté rue du Carpon: implantation } \\
\text { d'habitat dans un parc. Conservation } \\
\text { du mur }\end{array}$ & $\begin{array}{l}\text { Côté rue du Carpon: implantation } \\
\text { de «villas urbaines". Conservation } \\
\text { du mur. }\end{array}$ \\
\hline $\begin{array}{l}\text { Cheminements } \\
\text { doux et espaces } \\
\text { publics }\end{array}$ & , par- & $\begin{array}{l}\text { Mail de traverse reliant Pontaniou aux } \\
\text { ateliers } \\
\text { Diagonale piétonne dans les ateliers }\end{array}$ & $\begin{array}{l}\text { Un mail public irriguant le projet sus- } \\
\text { ceptible d'accueillir le tramway }\end{array}$ \\
\hline $\begin{array}{l}\text { Accessibilité et } \\
\text { stationnement }\end{array}$ & $\begin{array}{l}\text { Grand parking mutualisé } \\
\text { Voirie de desserte et de liaison en } \\
\text { front de rivière pour relier au fond de } \\
\text { Penfeld }\end{array}$ & $\begin{array}{l}\text { Parking dans l'atelier électricité } \\
\text { conservé } \\
\text { Avenue structurante longitudinale }\end{array}$ & $\begin{array}{l}\text { Deux nouveaux franchissements : un } \\
\text { pont pour le passage du tramway + } \\
\text { circulations douces et une passerelle } \\
\text { piétonne et cycles }\end{array}$ \\
\hline $\begin{array}{l}\text { Coûts annoncés } \\
\text { (espace public } \\
\text { et bâtiments } \\
\text { reconvertis) }\end{array}$ & 41,9 millions d'euros & $\begin{array}{l}1,1 \text { millions d'euros (+132 millions } \\
\text { our les bâtiments neufs) }\end{array}$ & Non précisé \\
\hline
\end{tabular}

Tableau 1 : Synthèse des trois projets d'urbanisme en concurrence (source : Madec, 2005 ; Fortier, 2005 ; Reichen et Robert, 2005)

Summary of the three urban projects in competition

L'intention n'étant évidemment pas ici de défendre un projet plutôt qu'un autre, le tableau joint aide tout de même à clarifier concepts et partis d'urbanisme défendus par les trois équipes, avec un certain nombre de points de convergence, bien qu'au final, les réponses se soient révélées assez contrastées (tableau 1). Les trois équipes ont dû concevoir le projet en tenant compte de la définition, en parallèle du marché de définition, du tracé du futur tramway. Ce paramètre a conduit à des scénarios prudents pour les équipes Fortier et Robert, tandis que l'équipe Madec fait du projet d'infrastructure la colonne vertébrale du projet urbain conduisant à deux propositions qui 
furent jugées non conformes à la commande politique par le comité de pilotage : la proposition d'un nouveau pont sur la Penfeld accueillant le tramway et la projection d'une station du tramway dans les grands ateliers. Alors même que la démarche environnementale est la plus développée des trois propositions d'urbanistes au risque de constituer une stratégie de séduction trop visible, voire utopique et irréaliste selon certains ${ }^{12}$, le « projet Madec » est ainsi rejeté par les élus crispés par cette double proposition autour du tramway.

Une tension est observable lorsque l'on se penche sur la commande d'une part, et les réponses des urbanistes d'autre part. La première se construit autour du couple contextualisé/décontextualisé. Dans le cas brestois, à une commande qui vise à révéler et à s'ancrer dans une soi-disante « identité brestoise », le «projet Madec » répond par un récit global : « De nos jours, tout projet urbain est confronté à la dégradation régulière des conditions de vie en ville, et à l'impact majeur de la conception des villes sur la crise planétaire, notamment pour le dérèglement climatique. Aussi notre équipe propose pour le futur quartier des Capucins, un projet qui s'attache à améliorer les conditions de vie à Brest et à ne pas dégrader davantage notre planète» (Madec, 2005). Si l'on veut bien croire que le point d'équilibre qui résout la tension serait le récit "glocalisé », au croisement d'enjeux globaux et locaux, que penser de cette nouvelle affaire qui survient en juin 2005 lorsque l'association Mécénat Bretagne fait état de son souhait de voir François Pinault qui s'est alors désengagé de l'île Seguin, s’intéresser à Brest pour ses collections? Et de fait, le maire François Cuillandre écrit en ce sens à l'intéressé au cours de l'été 2005. Bilan de l'affaire révélé par l'élu à la presse locale : «Il m'a répondu poliment pour me dire qu'il privilégiait le palais Grassi de Venise (!), mais n'a cependant pas totalement fermé la porte » (Le Télégramme de Brest, 15 septembre 2005)...

\section{Mises en public et renégociation de "l'identité brestoise ${ }^{13}$ »}

Le temps du marché de définition constitue une véritable entrée en scène(s) de la Penfeld et du plateau des Capucins. Événement oblige, comment le projet est-il rendu public ? À cette publicisation naissante, s'ajoute l'épreuve de la participation citadine évoquée rapidement plus loin.

\section{L’ENTRÉE EN SCÈNE(S) DU PLATEAU DES CAPUCINS}

En lançant la procédure, les élus ont commandité des récits d'anticipation de la part des trois équipes mobilisant de nouvelles images du site. Pendant le temps de cette procédure, d'anciennes images ont été également réactivées (l'époque d'avant, lorsque ce plateau était occupé par un monastère). Jamais autant de discours (oraux, textuels, iconographiques) enthousiastes n'ont été livrés sur ce cœur de ville interdit. Le travail de reconversion symbolique est en cours et la visibilité inédite donnée à ce territoire à réinvestir trouve ici une charge émotionnelle singulière étant donné l'absence de « prises » pour les habitants (Cozic et Granvaud, 2004). La magie du marketing territorial se traduit par la production de mythes mobilisateurs ( faire de Brest une référence en terme de développement durable»), de concepts alléchants (une «Villa Médicis Océane » dans la prison désaffectée!), d’objets spatiaux ( « cours des Capucins », " grand mail », " place haute des excellences brestoises ») qui viennent innerver le plateau. Une telle vision globale vient de fait rompre avec les représentations de la Marine dont les cartes ignorent superbement la ville : le fil conducteur pour cet acteur, c'est la Penfeld et l'enceinte de la base navale. Le discours du Capitaine de vaisseau Cavelier, chargé de mission auprès de l'adjoint territorial au commandant, est sans équivoque : "L'espace de la Marine est d'abord un outil de production protégé juridiquement

12. Dans l'entretien réalisé en juin 2007 avec Annick Cléach, Vice-Présidente de BMO, c'est le sentiment qu’elle a gardé du «projet Madec » : «On avait dit au départ de travailler les deux options (avec ou sans nouveau pont). Puis très vite, avant même la fin du marché, on a dit que l'on demandait d'abandonner l'option pont. Madec a refusé de nous entendre. Qui plus est, son projet était utopique. »

13. Les guillemets s'imposent. Le mot d'identité est mobilisé par les acteurs locaux eux-mêmes. 


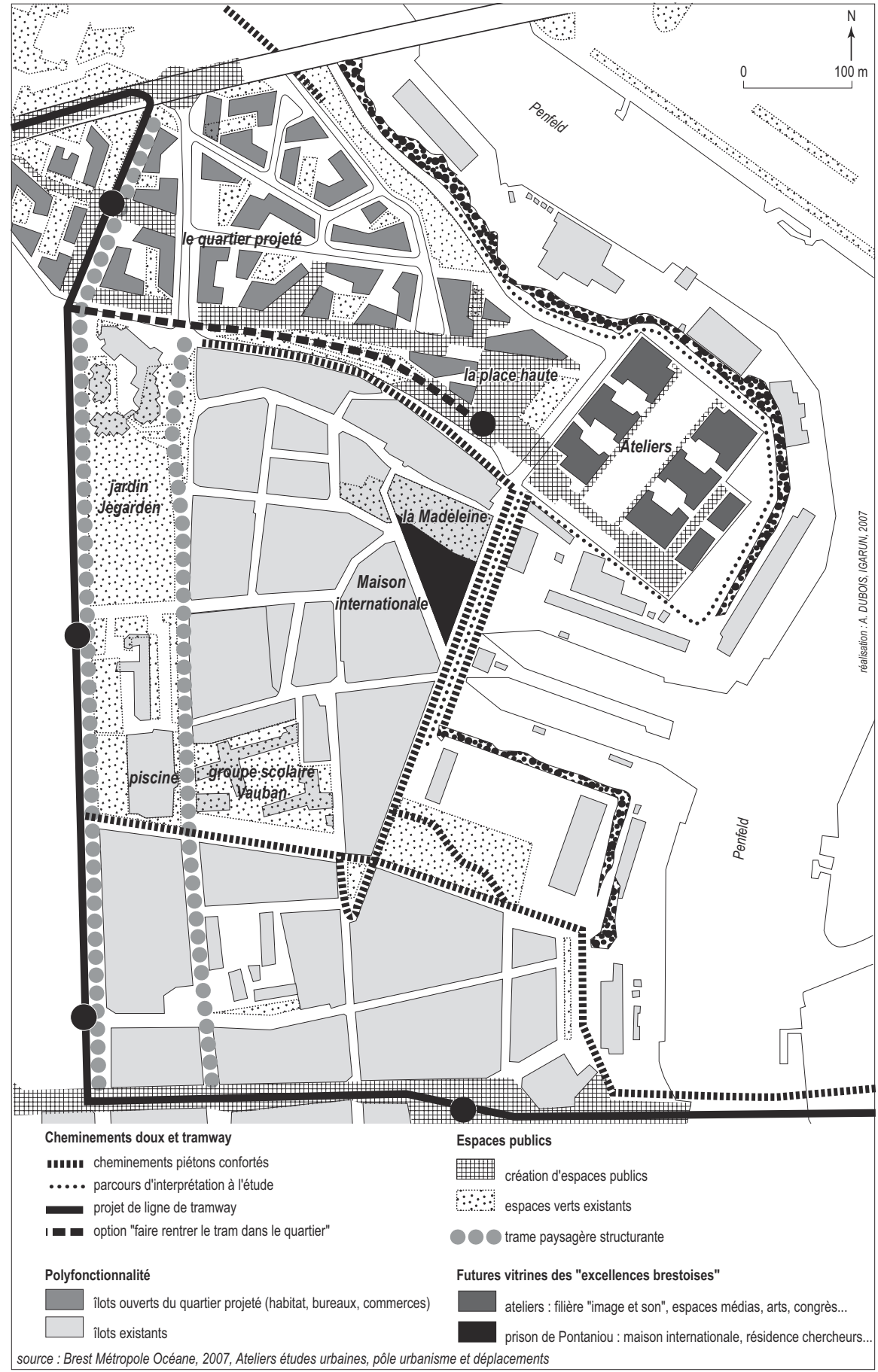

Figure 3 : Le « projet Fortier » : situation en 2007 (source : BMO, 2007, Atelier études urbaines, pôle urbanisme et déplacements)

Fortier's project in 2007 
par son statut de domaine public. Les terrains ne sont pas classés en fonction de leur utilité ou de leur inutilité, mais selon leur rattachement à une branche d'activité : centre de formation, atelier des bâtiments en fer, atelier des locomotives. Il n'y a pas de vision d'ensemble du site, mais une vision fragmentée. Un espace laissé vacant un temps peut avoir un autre usage productif : donc la friche peut être temporaire pour nous » (entretien réalisé en juin 2005) ${ }^{14}$.

En lien avec le travail des urbanistes, la mise en projet du plateau s'accompagne de la démultiplication des scènes publiques où l'on parle de l'aménagement du site, mais aussi de l'histoire des lieux. Sans être exhaustif, on peut distinguer des scènes locales et des scènes extérieures hors de Brest. Au niveau local, le projet a initié la création de sites ou pages Internet dédiés à la Penfeld. BMO a construit en 2005 une page web consacrée aux trois sites de projet localisés sur la Penfeld (www.cub-brest.fr/penfeld). Peu élaborée, la page est purement informative sur le plateau des Capucins, donne des liens qui renvoient aux projets des trois équipes, aux numéros de Sillage (la revue de $\mathrm{BMO}$ ) qui ont évoqué le dossier. Elle donne également accès à des images d'archives du site. Sur le forum début 2007, on a recensé 15 messages de réactions spontanées au projet. Le site Internet de l'association Rue de Penfeld née en 1995 (www.penfeld.net) est plus riche d'informations. Comptes rendus des rares débats publics organisés par l'association («Penfeld sur son 31 » en 2003 et 2004), interviews de personnalités brestoises, espace forum et même jeux proposés (un jeu de l'oie autour des rives de la Penfeld). Le ton peut être assez potache (comme ce slogan savoureux : « La chance aux chansons, la Vénus à Milo, la tarte à la crème, la Seine aux Parisiens, la Penfeld aux Brestois »). À travers les articles parus en nombre entre 2002 et 2004, les membres de l'association ont surtout cherché à interpeller et à trouver leur place dans le débat sur le plateau des Capucins (Rue de Penfeld, 2004). Depuis 2005, le débat public s'est tari assez brutalement et a glissé vers d'autres sites Internet plus politisés et non consacrés uniquement à l'aménagement de la Penfeld - par exemple le site des Verts (www.brestowvert.net) ou celui de l'association de la rue Saint-Malo (www.vivrelarue.net). Les pétitions circulent et les avis polémiques sont plus tranchés sur les différents projets ${ }^{15}$. Enfin, les articles de la presse locale à propos des Capucins ont répercuté l'actualité du dossier façon résultat d'un match de foot (la une du Télégramme de Brest du 29 septembre 2005 était : "Fortier 5 voix, Madec 1»), et ont fait circuler les images de synthèse et autres plans des équipes d'urbanistes. Une lecture rapide des articles du quotidien Le Télégramme de Brest laisse penser que l'information est descendante, issue des conférences de presse et autres dossiers fabriqués par le service communication de BMO. Certains articles ne sont qu'un décalque de l'information diffusée dans la revue de BMO.

La montée en puissance du projet passe par le nécessaire dépassement des scènes locales. Aussi le projet est-il montré depuis la fin du marché de définition dans un réseau de lieux et d'événementiels composant le parcours devenu incontournable pour qu'une opération d'aménagement acquière une stature de "grand projet ». Le projet des Capucins figure ainsi parmi les 50 projets de l'édition 2006 du Forum des projets urbains organisé chaque année par Innovapresse. Toujours en 2006, le projet figure également dans le «spécial aménagement » annuel de la revue Le Moniteur. Trois pages présentent les grandes orientations du "projet Fortier », auxquelles s'ajoutent une interview du maire de Brest, ainsi qu'un témoignage de l'urbaniste lauréat. En cela le projet est à peine sorti du marché de définition qu'il est aspiré dans une logique de mise sur le (grand) marché des projets urbains qui comptent en France. En parallèle, BMO a lancé une seconde page web dédiée aux investisseurs (www.cub-brest.fr/investinbrest). Le guide de l'investisseur renseigne

14. Un parallèle est à faire sur la représentation de la friche par la Marine et par le monde ferroviaire qui sont dans les deux cas très différentes de celle des aménageurs et des élus. Pour ces deux grands propriétaires fonciers, il n'y a pas une forte incitation en interne pour rationaliser les occupations et mettre en œuvre une politique d'arbitrage des actifs destinée à dégager des terrains mutables. La donne est sans doute en train de changer depuis le début des années 2000 (cf. exposé d'O. Danziger, RFF - Direction du patrimoine, «RFF : Quels partenariats pour la régénération des friches ferroviaires », Rencontres européennes des friches « Revit » à Nantes le 2 février 2006). Voir aussi J. Dubois-Maury, 2004.

15. Le «projet Fortier » est par exemple qualifié de "verrue libérale dans un écrin que représentait cet espace à investir » (C. Perrot, «Gauche alternative et citoyenne », [www.brestouvert.net $]$ ). 
sur l'offre en immobilier de bureaux (prix pratiqués, démarches d'accompagnement) et sur les grands aménagements brestois du moment (port du château, nouvelle aérogare, salle des musiques actuelles, tramway, rénovation du centre-ville et plateau des Capucins).

À la lumière de ce corpus composite que nous avons collecté, la figure du maire de Brest (également président de BMO) est le pilier attendu du « portage communicationnel » de l'aménagement. À ce titre, la désignation même du projet est un enjeu politique et marketing à part entière. BMO et son président parlent désormais non plus de la Penfeld, mais de "l'Espace Penfeld » pour évoquer les trois sites du Château, des Capucins et du Fond de Penfeld. L'élu doit porter la bonne nouvelle sur le front de scènes publiques qui se sont démultipliées depuis 2005 : «Ce site est aussi exceptionnel en Europe. Je parle bien d'une dimension européenne. Je souhaite que chacun prenne la mesure des travaux qui seront engagés ici, comparables à ceux des grandes cités européennes telles que Barcelone, Cardiff ou Venise. C'est donc bien l'un des grands projets de notre ville pour les 10 prochaines années et il me paraît important de le considérer avec fierté » (discours du maire, 2 juin 2005, lors de la présentation des trois projets aux Brestois).

\section{PARCOURS ET CENTRE D'INTERPRÉTATION OU COMMENT NÉGOCIER LA QUESTION IDENTITAIRE}

Décidé dès avant le marché de définition au cours des réunions pilotées par la mission Penfeld, l'idée d'un parcours et d'un centre d'interprétation est validée courant 2004 suite à la phase ouverte du marché de définition. Le document-programme à l'issue de cette phase évoque la pertinence d'« un lieu dédié de compréhension du site et de la ville par thématiques », mais aussi « lieu d'expression des excellences locales et centre de ressources de la mémoire vive » (CUB, 2004). Un groupe de réflexion s'est ainsi constitué et réuni à plusieurs reprises de mars à juin 2005 en parallèle de la phase fermée.

Le temps du projet met les acteurs brestois dans l'obligation d'avoir à penser la présentation de leur ville sur ce territoire stratégique et symbolique. Double est l'intérêt qu'il y a à suivre les discussions du groupe de travail qui a réuni des universitaires locaux reconnus (Daniel Le Couédic, directeur de l'Institut de Géoarchitecture et chercheur de renom en architecture et urbanisme, Philippe Jarnoux, historien), des élus (dont Annick Cléach), des professionnels des pôles Développement et Urbanisme de la communauté urbaine et des cadres de la conservation (musée du Beaux-Arts, Archives communautaires, Commission du Patrimoine de la Marine nationale). La mise en débat de la question identitaire se traduit par deux questionnements qui sont liés. Comment éviter le piège du localisme d'une histoire ô combien atypique de la ville ${ }^{16}$ ? Et comment dépasser «la présence du monumental atelier des machines qui fonde a priori la vocation des Capucins à être l'interprète du patrimoine et de l'histoire de Brest » (CUB, 2005) ? Un débat est ainsi ouvert sur les lieux ${ }^{17}$ (patrimoniaux ou non) qu'une collectivité (se) désigne comme dédiés à l'identité et à l'histoire de la ville ${ }^{18}$.

À Brest, la réflexion n'a semble-t-il pas encore donné lieu à une stratégie stabilisée. Le groupe paraît chercher une voie qui permettrait de déminer un legs historique (post-Seconde Guerre mondiale) qui a généré le désamour des habitants pour leur cité depuis la Reconstruction. Même si la question de l'intégration et du statut de l'objet spatial « grands ateliers » dans la ville ne sera pas réglée une fois pour toutes au cours de ces réunions, le groupe de travail se constitue en force de proposition pour

16. Rappelons que «Brest même a été détruite deux fois, de deux façons différentes : par les armes et par un choix d’urbanisme qui conduisit à l'arasement systématique de ses ruines comme de ses fortifications et à la constitution d'un plateau artificiel, parfois trente mètres au-dessus du site initial » (Le Couédic in Le Gallo Y. et al., 1992).

17. Non seulement le contenant, mais aussi le contenu de ces lieux scénographiés sont déterminants dans la mise en scène identitaire et patrimoniale de la ville.

18. D'une ville à l'autre, les modes de présentation sont de fait très divers : si certaines villes se donnent à voir sur un mode extrêmement auto-centré (exemple à nouveau de Nantes et le musée flambant neuf de la Ville de Nantes accueilli dans les locaux du Château des Ducs de Bretagne), d'autres villes jouent davantage sur l'articulation à un territoire environnant d'identification plus vaste (exemple du musée de Bretagne hébergé dans le récent bâtiment culturel « Les Champs Libres » à Rennes). 
faire valoir le concept de « ville-laboratoire » comme cœur du projet et faire de la ville elle-même un parcours et un objet d'interprétation. D’après D. Le Couédic, l'idée serait d'articuler le local et l'universel à travers le prisme de l'histoire de Brest, mais aussi d'ouvrir aux enjeux contemporains de la ville « en train de se faire » $(\mathrm{CUB}, 2005)$. Des pistes de mise en œuvre sont ainsi repérées ${ }^{19}$ : concevoir une exposition permanente autour de trois phases de l'histoire de Brest (la ville nouvelle du $\mathrm{XVII}^{\mathrm{e}}$ siècle, la ville reconstruite du XX ${ }^{\mathrm{e}}$ siècle et « la quête d'un surcroît d'âme [?] à l'aube du XIX »), développer un concept de réseau de lieux identitaires et patrimoniaux à travers la ville, créer un forum permanent où les projets brestois seront présentés et débattus de manière à systématiser la mise en public de la fabrique de la ville via la confrontation des élus et des Brestois, mettre en place un Groupe d'Intérêt Scientifique fédérant chercheurs et archivistes. En 2007, un premier tronçon du parcours d'interprétation est en cours d'élaboration depuis le jardin des Explorateurs situé en face du Château jusqu'aux ateliers. Mais aucun tracé n'est encore officiel.

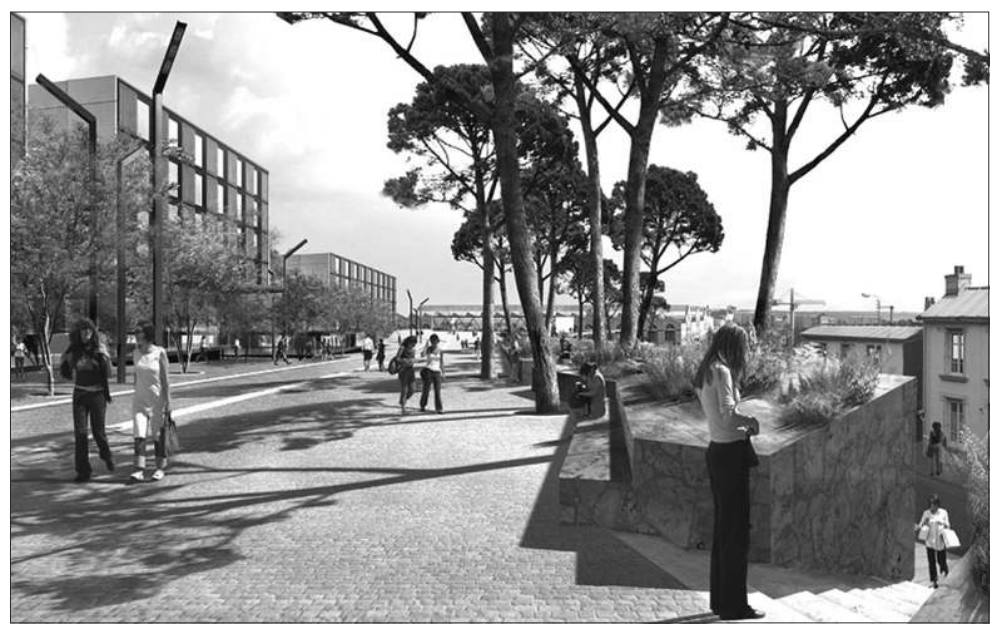

Photo 2 : La future promenade projetée (source : Fortier, 2005) project

The public walk in

\section{Urbanisme « négocié » : le mirage de la «vision partagée »?}

D’après la mission Penfeld, l'objectif d'une vision partagée de l'évolution de la Penfeld dans les 10 à 20 ans à venir a été atteint au moment où celle-ci a remis son bilan. Qu'en est-il vraiment? Le marché de définition a été lancé alors même que les questions de maîtrise foncière ne sont pas réglées avec le ministère de la Défense ${ }^{20}$. Il n'y a point là de spécificité au cas brestois, si l'on veut bien se rappeler d'autres expériences similaires de gestation de projet qui mettent aux prises une maîtrise d'ouvrage à de grands propriétaires fonciers publics. Dans cette logique, la formalisation de desseins d'urbanisme vient se heurter à une double difficulté liée à la maîtrise foncière. Tout d'abord, le principe de cession des Capucins a été validé par le ministère de la Défense depuis 2004, tandis qu'il reste en 2005 seulement « envisageable » pour le plateau de Quéliverzan. En 2007, le principe de cession est acquis pour les deux parties du projet. La négociation se joue aussi entre la Mission de Réalisation des Actifs Immobiliers (MRAI) du ministère de la Défense et BMO pour déterminer le prix de cession. Un accord négocié serait en passe d'être trouvé :

19. À noter que l'exemple du Havre n'est pas convoqué par les acteurs et membres du groupe de réflexion (cf. pour l'analyse de la situation havraise, M. Gravari-Barbas, 2004). Cf. aussi V. Veschambre, 2005.

20. « La marine nationale n'est pas demandeuse de cette opération, mais s’y rallie volontiers pour permettre à la communauté urbaine de réaliser ses projets. [...] La marine est ouverte à la discussion. » (extraits de la lettre du vice-président d'escadre Laurent Mérer à Gérard de Senneville, chargé de mission Penfeld, 25 juin 2004). 
« c'est la politique des petits pas, mais on avance », nous a confié Annick Cléach, Première VicePrésidente de BMO, en juin 2007.

Jusqu'aux débuts aux années 1990, le ministère de la Défense s'est refusé à intervenir au stade du réaménagement de ses biens dont il n'a plus l'utilité (Dubois-Maury, 2004). Le dessaisissement pour Euralille ou La Part-Dieu à Lyon s'est ainsi fait sans le ministère de la Défense. La rupture est actée dès la création de la MRAI en 1987 qui engage souvent des études préalables de faisabilité pour être en mesure de négocier les cessions le plus avantageusement possible. La mutation des terrains militaires correspond au plan juridique à trois étages successifs de dérogations au droit commun (Leroux, 2003) : tout d'abord, le rattachement des produits de cession au budget du ministère de la Défense, et non au budget de l'État (depuis 1984), ensuite, une autonomie du ministère de la Défense dans le choix des bénéficiaires des cessions (depuis 1986), enfin une large autonomie en matière de fixation des prix de cession (protocole de 1987 complété par un additif de 1995). À l'échelle nationale, la stratégie du ministère de la Défense devient comparable à celle conduite par l'établissement public Réseau Ferré de France (RFF). Afin d'optimiser les ventes de foncier pour gérer la dette de la SNCF, ce dernier cherche à être intégré très amont pour connaître et même participer aux utilisations futures. Objectif : en se mettant d'accord en amont avec la collectivité sur les programmes qui conditionnent pour une large part la valeur des terrains, les difficultés concernant le prix du foncier sont déminées. À Brest, et même si elle n'est pas apparue comme force de proposition, la MRAI s'est occupé de l'évaluation économique des projets des trois équipes afin logiquement de déterminer son prix en fonction des ventes potentielles de charges foncières par le futur aménageur et des dépenses (dépollution, etc.). Dans l'affaire du plateau des Capucins, la négociation est en cours depuis 2005 et BMO a déjà tenté sans succès de faire vibrer la corde sensible en demandant le foncier à un euro symbolique au titre de la dette du ministère de la Défense vis-à-vis de Brest, au titre des fortes suppressions de postes à l'arsenal depuis 1992.

À cette tension État/local s'ajoutent des difficultés rencontrées par BMO. De l'avis d'une chargée de mission de l'Agence d'urbanisme de Brest, « la culture de projet avec les compétences en ingénierie et management essentielles pour intégrer les opérations lorsqu'elles sont à tiroirs sont encore très récentes au sein de BMO » (entretien réalisé en juin 2006). De plus, la confrontation des habitants aux élus et professionnels publics et privés de l'aménagement ne s'est pas réellement concrétisée durant le marché de définition. Y a-t-il eu restriction volontaire de l'épreuve de la participation ou bien réel manque de savoir-faire de l'intercommunalité? En décembre 2003, lors d'une réunion publique d'informations sur l'opération de renouvellement urbain du quartier de Recouvrance, Annick Cléach, vice-présidente de BMO, a reconnu avec honnêteté que la communauté urbaine ne savait pas organiser de concertation avec la population et envisageait de faire appel au cabinet parisien Attitudes Urbaines afin d'animer cette phase de démocratie participative qui se révéla au final extrêmement limitée. Aucun dispositif participatif n'a en effet été prévu durant la phase ouverte, et ce n'est qu'au moment de la phase fermée que des expositions des projets des trois équipes ont donné lieu à des situations d'interaction entre élus, professionnels et habitants inédites jusqu'alors. En somme, le discours officiel sur la vision partagée semble cacher un certain nombre d'asymétries.

Datée du 24 août 2005, la lettre ouverte de Marc Wiel au maire de Brest, urbaniste de renom, ancien directeur de l'Agence d'urbanisme de Brest, est une charge contre la conduite du marché de définition par les acteurs brestois. Pêle-mêle, il dénonce la fragilité, voire l'inconsistance du programme, le manque d'études préalables et d'une vraie concertation avant le lancement du marché. La question d'un nouveau pont a, selon l'urbaniste, généré des crispations prématurées autour d'un lien sur lequel aucune concertation préalable n'a eu lieu. Ce piège aurait ainsi coûté cher au «projet Madec ${ }^{21}$ ». Quant à la réflexion sur le devenir des ateliers, une étude de programmation initiale aurait clairement manqué et, les trois équipes y auraient, selon lui, projeté des combinaisons plus ou moins aléatoires d'espaces imbriqués correspondant à des éléments

21. En 2006, les Verts ont lancé un appel à signature sur le « désir de pont-passerelle ». 
de programme non stabilisés par le commanditaire. De façon polémique, Marc Weil souligne les difficultés pour la maîtrise d'ouvrage brestoise à penser le provisoire et à le combiner avec des usages définitifs. Au sujet des grands ateliers, le propos de l'urbaniste est sans équivoque : «Avoir un tel espace couvert est une chance phénoménale pour Brest; cela suppose une programmation des espaces ouverte à des usages éphémères des lieux. Et, il ne faut plus raisonner comme pour une opération qui se boucle en quelques années. » Il est assurément difficile de cerner la part de vérité du propos de Marc Wiel. Certaines accusations nous ont paru assez rudes, d'autant que les élus eux-mêmes semblent conscients que « les opportunités de la prochaine décennie ne sont par définition pas programmables ni prévisibles » (cf. entretien avec Annick Cléach, juin 2007). Au final, entre les lignes de la polémique, on peut y lire, d'une part, la démarche exploratoire de BMO lancée pour un projet d'envergure qui ne doit pas par définition être figé à son lancement, d'autre part, des fixations parfois prématurées de la part des urbanistes en concurrence et qui montrent toute la difficulté pour le commanditaire à trouver un point d'équilibre sur le degré de définition à ce stade pré-opérationnel.

\section{Conclusion : le renouvellement urbain dans l'Ouest français, circulations et convergences}

Brest Métropole Océane joue gros - aussi bien les élus que les cadres techniques - à travers le projet du plateau des Capucins dans l'enjeu d'une métropolisation à conquérir. L'acculturation en cours du milieu brestois à la culture du « grand projet urbain » est double puisqu'elle impose de régler et le portage politique et le pilotage technique dans un contexte plein d'incertitudes au moment du marché de définition (négociations du foncier, devenir des grands ateliers, tracé du tramway). Le succès de la Mission Penfeld est sans doute réel dans l'instauration d'un dialogue sur les destinations et les programmes entre la communauté urbaine et le grand propriétaire foncier (la Marine et la MRAI qui souhaite optimiser la valorisation de ses biens). Mais ce serait aller vite en besogne d'en conclure à « une vision partagée » : les asymétries sont nombreuses et BMO négocie ferme auprès du ministère de la Défense qui ne s'en laisse pas facilement compter. Enfin, depuis 2006, ce projet a été rapidement incorporé à la stratégie métropolitaine au point de constituer l'un des emblèmes de l'action territoriale de Brest Métropole Océane. La «traduction » de cette montée en puissance de l'intercommunalité s'effectue sur différentes scènes locales et externes. Le projet urbain lui-même a acquis le label de « grand projet » en France par le parcours de promotion que les acteurs locaux lui ont fait suivre dans différents événementiels. Dès ce stade de l'action collective, l'on observe une démultiplication du projet en micro-objets et autant de « dossiers » et «affaires » à suivre : le pont au-dessus de la Penfeld (objet-lien en discussion); le parcours et centre d'interprétation (ou comment résoudre la question identitaire de Brest); les nefs des grands ateliers (objet attracteur appelé à devenir un haut lieu de culture).

Le projet brestois invite à questionner les manières de faire du renouvellement urbain de friches dans les grandes villes de l'ouest français. Un rapide passage en revue des projets « Île de Nantes » à Nantes, «Ville-Port » à Saint-Nazaire, «Docks Vauban » au Havre, " Mayenne-Capucins » à Angers, «La Courrouze » à Rennes ${ }^{22}$, conduit à faire état d'un fort nombre de convergences qui traduisent des circulations fortes et des effets de modèles.

La circulation de «bonnes pratiques » urbanistiques est un fait remarquable. À Brest, la commande d'un outil «plan-guide » met sur la piste du «projet Chemetoff », du nom du lauréat du marché de définition du projet « Île de Nantes », dont l'innovation a été identifiée et consacrée par les professionnels du ministère de l'Équipement (Masboungi, 2003) comme étant une «bonne pratique » en matière de pilotage d'un projet de renouvellement urbain en raison

22. Hormis le projet du Havre, tous ces projets sont référencés dans la plaquette de présentation de l'espace métropolitain Loire Bretagne. Il s'agit d'une coopération renforcée entre Nantes, Brest, Saint-Nazaire, Angers et Rennes qui existe depuis février 2005 et qui fait suite à l'appel à coopération métropolitaine lancé par la DIACT. 
de sa flexibilité et de sa réactivité ${ }^{23}$. Les options retenues pour le projet brestois du plateau des Capucins sont également le reflet des principes qui font actuellement recette dans une bonne part des "grands projets » urbains : mixité fonctionnelle, mixité sociale, démarche "Haute Qualité Environnementale», intégration forte aux politiques de transports collectifs. L'ancrage de la réflexion sur un temps long (au-delà de la durée du mandat électoral), l'acculturation à une transversalité et la coordination entre les différents services techniques des collectivités et la structure aménageur font partie des pré-requis dans la conduite de l'action collective (Bonneville, 2004). Plus précisément encore, une circulation d'un certain type d'options programmatiques est particulièrement prégnante. Au plan économique, deux enjeux sont mobilisés fortement : le premier autour du développement d'une économie culturelle, commerciale et touristique, le second autour du renforcement de «l'économie de la connaissance ${ }^{24}$ » fondée sur le développement de pôles spécialisés sur des segments productifs tels que les médias, la santé, l'environnement, ou encore la formation. D'une ville à l'autre, les acteurs privilégient l'une des deux économies ou bien couplent économie de la connaissance et économie culturelle et touristique. Dans le projet «Ville-Port» de Saint-Nazaire, le parti d'une centralité culturelle forte qui procède à la fois de créations de lieux culturels (multiplexe, musée Escal'Atlantique, lieu des formes émergentes «Life » et scène des musiques actuelles) et de relocalisations (salle de concerts «VIP » et théâtre) est très clairement repérable et déployée au travers de synergies public/privé. Le projet « Île de Nantes » permet d'identifier une stratégie semblable autour du levier culturel et touristique avec la mise en place des « Machines de l'Île » (dont l'éléphant de Royal de Luxe) de l'événement artistique « Estuaire 2007 »(Devisme, 2006 ; Chasseriau, 2004). Dans ce projet, l'aménageur articule l'approche culturelle et touristique avec une stratégie de créer des pôles de compétences (biotechnologies, éco-technologies, santé, et formation avec la relocalisation de l'école d'architecture et le futur «campus des arts »).

Culture (sous toutes ses formes, institutionnelle, marchande...), événementiels et activités «créatives » qui traduisent l'injonction faite par l'État aux collectivités locales de déployer des pôles de compétitivité occupent une place de choix dans l'esprit des acteurs qui cherchent à mobiliser des réseaux professionnels et des ressources cognitives multiformes. La diffusion de démarches étrangères semble être une piste possible dans l'appréhension de la circulation des options de programme. L'exemple barcelonais est naturellement incontournable : la démarche y est particulièrement connue dans le domaine de la requalification des différents segments de son front de mer (Rodrigues-Malta, 1999). Elle est sans doute toute aussi pionnière dans le projet «Barcelona 22@» localisé dans le quartier Poble Nou (situé au sud du prolongement de la Diagonal vers la mer), qui a pour finalité de régénérer le tissu de friches industrielles du « Manchester catalan » en « district de l'innovation » polarisé autour de quatre clusters (média, biomédical, TIC et énergie) ${ }^{25}$.

Toutefois, la déclinaison de ce socle de principes et autres « bonnes pratiques » prend des voies qui se révèlent beaucoup plus diverses que l'on aurait pu le penser de prime abord. Un premier type remarquable est le projet urbain qui couple renouvellement urbain et développement durable. Les acteurs vont jusqu'à labelliser le territoire d'« éco-quartier » par exemple à Nantes ou à Angers ${ }^{26}$. Un second type de projet concrétise plus fortement le parti d'une centralité à haute qualité urbaine. Le projet «Docks Vauban » au Havre sur 17 ha est un exemple d'action urbaine qui traduit un morceau de ville à haute qualité (équipements publics culturels et de loisirs et

23. Outil technique actualisé chaque trimestre, le plan-guide permet à l'aménageur d'intégrer de nouvelles opérations et hypothèses de constructibilité au fil des opportunités initiées par les acteurs publics et le marché.

24. L'économie de la connaissance est pensée comme une économie «présentielle » d'archipel (pour reprendre les propos de P. Veltz). Cf. n 101 des Annales de la Recherche Urbaine, «Économies, connaissances, territoires » publié en 2006. Voir aussi T. Nghiem, 2006).

25. Notre propos fait suite à un voyage d'études effectué à Barcelone en avril 2007. Cf. [www.22@barcelona.com]. Pour une approche européenne, cf. Pinson, 2006.

26. Cf. V. Veschambre, 2006. 
commerces) ciblé pour ménages aisés. Le marketing assuré par l'aménageur véhicule un discours sans détours : «En plein cœur du Havre, sur un site historique entièrement réhabilité - les docks portuaires du XIX ${ }^{\mathrm{e}}$ siècle - Docks Vauban sera un véritable retail park ${ }^{27}$, combinant les atouts d'un site de périphérie à dimension régionale et l'attractivité d'un centre commercial urbain ancré dans l'histoire. Docks Vauban s'intègrera dans un ensemble d'équipements à fort pouvoir d'attraction (Docks Océane, Docks Cafés, Centre de la Mer, complexe aquatique, casino...) pour proposer une offre commerciale forte et qualitative, à la fois permanente et temporaire, à une population mixte d'habitants et de résidents secondaires dotée d'un fort pouvoir d'achat ${ }^{28}{ }$. Un troisième type correspond aux projets qui absorbent tous les enjeux : développement économique (mise en place de clusters, priorité à l'économie culturelle et touristique, renforcement et création de centralités commerciales), approche environnementale de l'urbanisme et construction d'une « offre urbaine » structurée par des espaces publics de qualité. Le projet « Île de Nantes » est un cas caractéristique de cumul des stratégies.

Au final, l'aménagement du plateau des Capucins à Brest illustre bien les emprunts et les circulations qui s'opèrent dans l'univers des projets urbains. L'identification des convergences dans les manières de faire et des modèles reste à approfondir pour nourrir une théorie du renouvellement urbain appliquée aux grandes villes du Grand Ouest français.

\section{Sources}

AdeupA, 2003. - Étude sur l'évolution et les enjeux des ports et du front de mer, Rapport d'étude, Anne Grasset, Brest, 40 p. + annexes.

Cozic (J.), Granvaud (M.-L.), 2004. - Face au déficit symbolique, un désir d'appropriation. Référentiel des représentations spatiales des quartiers de Pontaniou et de Recouvrance, Travail étudiant encadré par P.-A. Barthel, Brest, Université de Bretagne Occidentale, 35 p.

CUB, 2005. - Les Capucins : parcours et centre d'interprétation, Brest, Compte rendu des réunions du groupe de travail de mars à juin 2005, 28 p.

CUB, 2004. - Document-programme pour l'aménagement du plateau des Capucins à Brest remis aux 3 équipes de maîtrise d'œuvre à l'issue de la phase collective du marché de définition, Brest, CUB/SEMAEB, 40 p.

CUB, 2003. - Espace Penfeld - Plateau des Capucins : éléments de réflexion pour un programme, Brest, Groupe de travail des élus de la CUB, 45 p.

De Senneville (G.), 2005. - Bilan de la phase de démarrage de la mission d'étude Penfeld à Brest (2002-2005), Paris, Conseil général des ponts et chaussées, Rapport de synthèse, 117 p.

ForTier (B.), 2005. - Brest métropole Océane : Plateau des Capucins, marché de définition, Brest, SEMAEB/ Brest Métropole Océane, n.p.

Madec (P.), 2005. - Brest métropole Océane: Plateau des Capucins, marché de définition, SEMAEB/Brest Métropole Océane, Brest, n.p.

Reichen (B.), Robert (P.), 2005. - Brest métropole Océane: Plateau des Capucins, marché de définition, SEMAEB/Brest Métropole Océane, Brest, n.p.

Rue de Penfeld, 2004. - «Penfeld sur son 31 », Compte rendu de la réunion publique du 30 avril 2004, Brest, 4 p.

Semaeb/Brest Métropole Océane, 2005. - Marché d'étude de définition : aménagement du plateau des Capucins à Brest, Brest, commission d'appel d'offre spécifique du 22 mars 2005, Rapport, 20 p.

27. La stratégie commerciale du « Retail Park » prévoit un nombre d’activités planifiées selon les tendances de consommation moderne et la spécificité du marché local, ainsi qu'une zone de loisirs.

28. Bel exemple des métamorphoses de la problématique des centralités commerciales explorée en particulier par A. Gasnier $(2003,2006)$ ! 


\section{Bibliographie}

Bonneville (M.), 2004, « Les ambiguïtés du renouvellement urbain en France », Les Annales de la recherche urbaine, $\mathrm{n}^{\circ}$ 101, « Renouvellement urbain », p. 7-16.

Chasseriau (A.), 2004. - «Au cœur du renouvellement urbain nantais : la Loire en projet », Norois, $n^{\circ} 192$, p. 71-84.

DeVISME (L.) (dir.), 2006. - L'analyse pragmatique d'un projet urbain : la mise à l'éprewve du plan-guide en projet de l'île de Nantes, Rapport final de recherche, contrat PUCA échelles et temporalités, 215 p.

Dubois-Maury (J.), 2004. - « La participation des propriétaires au renouvellement urbain », Les cahiers $d u$ Gridauh, "Droit et politique de renouvellement urbain », Paris, La Documentation française, p. 81-98.

Dumont (M.), Andrieu (D.), 2006. - « Qualité urbaine et ville durable à l'épreuve du renouvellement urbain. L'exemple du Grand Projet de Ville Malakoff Pré Gauchet à Nantes », Norois, nº 198, p. 7-20.

Gasnier (A.), 2006. - «Entre privatisation des lieux publics et publicisation des lieux privés », Urbanisme, $n^{\circ} 346$, Espaces publics, janvier/février, p. 70-73.

—, 2003. - « Saint-Nazaire, un renouvellement urbain inachevé », dans Risques, vulnérabilité et politiques de développement durable en milieu urbain, dans EMELIANOFF (C.) (dir.), Rapport de recherche, mi-parcours, contrat État-Région, vol. 2, Université du Maine, GREGUM/UMR ESO CNRS, p. 96-105.

Gravari-Barbas (M.), 2004. - «Patrimonialisation et réaffirmation symbolique du centre-ville du Havre. Rapports entre le jeu des acteurs et la production de l'espace », Annales de Géographie, n 640, nov-déc. p. 588-611.

Le Gallo (Y.), Henwood (P.), Henwood (A.), Berthou (C.), Champot (E), Le Couëdic (D.), Dieudonné (P.), Rouxel (F.), Wiel (M.), 1992. - Brest alias Brest. Trois siècles d'urbanisme, Liège, Mardaga, 237 p.

Leroux (P.) (dir.), 2003. - Présence et représentation de l'institution militaire dans la ville, Centre d'études en sciences sociales de la Défense, $261 \mathrm{p}$.

Masboungi (A.) (dir.), 2003. - La Loire dessine le projet. Île de Nantes, Paris, éditions La Villette/DGUHC, $192 \mathrm{p}$.

NGhiem (T.), 2006. - « Brest, une ville apprenante. La mutation d'une ville port militaire », Les Annales de la recherche urbaine, $\mathrm{n}^{\circ}$ 101, «Économies, connaissances, territoires », p. 134-137.

Pinson (G.), 2006. - «Projets de ville et gouvernance urbaine », Revue française de science politique, $\mathrm{n}^{\circ} 4$, vol. 56, p. 619-651.

Rodrigues-MaLta (R.), 1999. - «Villes d'Espagne en régénération », Annales de Géographie, nº 608, p. 397-419.

ThéVenot (L.), 2006. - L'action au pluriel. Sociologie des régimes d'engagement, Paris, éditions la découverte, $311 \mathrm{p}$.

Veschambre (V.), 2006. - « Patrimoine et développement durable : pléonasme ou contradiction? », Urbanisme, $\mathrm{n}^{\circ} 348$, mai-juin 2006, p. 57-60.

—, 2005. - « Le recyclage urbain, entre démolition et patrimonialisation : enjeux d'appropriation symbolique de l'espace. Réflexions à partir de quatre villes de l'Ouest », Norois, nº 195, p. 79-92.

Zetlaoui-Léger (J.), 2002. - «Marchés de définition simultanés : un succès à interroger », Urbanisme, juillet-août 2002, p. 14-17.

Cet article a été reçu le 22 mars 2007 et définitivement accepté le 20 juin 2007. 
